\title{
Acute glucose influx-induced mitochondrial hyperpolarization inactivates myosin phosphatase as a novel mechanism of vascular smooth muscle contraction
}

\author{
Jie Xu ${ }^{1,2}$, Hongyan Yang ${ }^{1}$, Lu Yang ${ }^{3}$, Zhen Wang ${ }^{1}$, Xinghua Qin ${ }^{1}$, Jiaheng Zhou ${ }^{1}$, Ling Dong ${ }^{1}$, Jia Li $\mathbb{D}^{1}$, Minsheng Zhu ${ }^{4}$,
} Xing Zhang (1) ${ }^{1}$ and Feng Gao (iD)

\begin{abstract}
It is well-established that long-term exposure of the vasculature to metabolic disturbances leads to abnormal vascular tone, while the physiological regulation of vascular tone upon acute metabolic challenge remains unknown. Here, we found that acute glucose challenge induced transient increases in blood pressure and vascular constriction in humans and mice. Ex vivo study in isolated thoracic aortas from mice showed that glucose-induced vascular constriction is dependent on glucose oxidation in vascular smooth muscle cells. Specifically, mitochondrial membrane potential $(\Delta \Psi \mathrm{m})$, an essential component in glucose oxidation, was increased along with glucose influx and positively regulated vascular smooth muscle tone. Mechanistically, mitochondrial hyperpolarization inhibited the activity of myosin light chain phosphatase (MLCP) in a $\mathrm{Ca}^{2+}$-independent manner through activation of Rho-associated kinase, leading to cell contraction. However, $\Delta \Psi \mathrm{m}$ regulated smooth muscle tone independently of the small G protein RhoA, a major regulator of Rho-associated kinase signaling. Furthermore, myosin phosphatase target subunit 1 (MYPT1) was found to be a key molecule in mediating MLCP activity regulated by $\triangle \Psi \mathrm{m} . \Delta \psi \mathrm{m}$ positively phosphorylated MYPT1, and either knockdown or knockout of MYPT1 abolished the effects of glucose in stimulating smooth muscle contraction. In addition, smooth muscle-specific Mypt1 knockout mice displayed blunted response to glucose challenge in blood pressure and vascular constriction and impaired clearance rate of circulating metabolites. These results suggested that glucose influx stimulates vascular smooth muscle contraction via mitochondrial hyperpolarization-inactivated myosin phosphatase, which represents a novel mechanism underlying vascular constriction and circulating metabolite clearance.
\end{abstract}

\section{Introduction}

Blood pressure (BP), one of the most commonly measured clinical parameters and one of the most commonly used indicators for cardiovascular health, is determined

Correspondence: Xing Zhang (zhangxing@fmmu.edu.cn)

${ }^{1}$ School of Aerospace Medicine, Fourth Military Medical University, Xi'an 710032, China

${ }^{2}$ Department of Cardiology, 986th Hospital, Fourth Military Medical University, Xi'an 710032, China

Full list of author information is available at the end of the article

These authors contributed equally: Jie Xu, Hongyan Yang

Edited by Q. Chen. mainly by the blood volume, cardiac output, and vascular resistance ${ }^{1-3}$. Among these factors, vascular resistance depends on a balance between contraction and relaxation of vascular smooth muscle cells (VSMCs) $)^{4,5}$. Besides mechanical stimuli, vascular smooth muscle tone is mainly regulated by vasoactive signals released from sympathetic nerves, endocrine organs, and parenchymal cells, including epinephrine, noradrenaline, angiotensin II, endothelin, NO, etc. ${ }^{4-6}$. Recent studies have shown that vascular smooth muscle tone is also regulated by metabolism $^{7-10}$. It is well-established that long-term exposure

\section{(c) The Author(s) 2021}

(c) Open Access This article is licensed under a Creative Commons Attribution 4.0 International License, which permits use, sharing, adaptation, distribution and reproduction cc. in any medium or format, as long as you give appropriate credit to the original author(s) and the source, provide a link to the Creative Commons license, and indicate if changes were made. The images or other third party material in this article are included in the article's Creative Commons license, unless indicated otherwise in a credit line to the material. If material is not included in the article's Creative Commons license and your intended use is not permitted by statutory regulation or exceeds the permitted use, you will need to obtain permission directly from the copyright holder. To view a copy of this license, visit http://creativecommons.org/licenses/by/4.0/. 
of the vasculature to metabolic disturbances leads to abnormal vascular tone ${ }^{7,8}$, while the physiological regulation of vascular tone upon acute metabolic challenge remains unknown.

The most abundant metabolite used by VSMCs is glucose. However, different from striated muscle cells (e.g., cardiomyocytes and skeletal muscle cells), VSMCs exhibit unusually high rates of glycolysis even under welloxygenated condition, relying on a large extent of ATP from glycolysis other than glucose oxidation to maintain their biological activity ${ }^{11}$. It is estimated that only $30 \%$ of the ATP supply comes from mitochondrial oxidation, and at least $90 \%$ of glycolysis resulting in lactate production ${ }^{12}$. In spite of the low contribution of mitochondria to the VSMC bioenergetics, several lines of evidence highlight the relevance of mitochondria in the regulation of VSMC function ${ }^{13}$. For example, mitochondria were reported to be involved in regulating VSMC contraction, proliferation, migration, and secretion; and mitochondrial dysfunction contributes to vascular pathologies such as atherosclerosis, stenosis, and hypertension ${ }^{10,13-15}$.

VSMC contraction is normally initiated by the increase of intracellular $\mathrm{Ca}^{2+}$ concentration, resulting in $\mathrm{Ca}^{2+} / \mathrm{cal}-$ modulin-dependent activation of myosin light chain (MLC) kinase (MLCK). MLCK phosphorylates MLC, allowing myosin to bind to actin filaments and thereby developing force and/or shortening cells ${ }^{4,5}$. In addition, MLC can be dephosphorylated by MLC phosphatase (MLCP), leading to cell relaxation. MLCP, a heterotrimer, is consisted of a catalytic type 1 of phosphatase subunit (PP1c $\delta$ ), a myosin phosphatase target subunit 1 (MYPT1), and a 20-kD subunit $^{5}$. MYPT1, the central regulator of MLCP, is mainly regulated by the small $\mathrm{G}$ protein RhoA and its downstream target Rho-associated kinase (ROCK). ROCK phosphorylates MYPT1, leading to the inhibition of MLCP activity and the resultant enhancement of MLC phosphorylation ${ }^{4}$. The other independent pathway involved in the regulation of MLCP activity is protein kinase $\mathrm{C}$-activated protein phosphatase-1 inhibitor of $17 \mathrm{kD}$ (CPI-17) ${ }^{16,17}$. CPI-17 behaves in a similar manner as MYPT1, whose activation inhibits MLCP activity. Recent evidence has shown that MLCP plays an important role in the regulation of vascular tone in both vascular health and diseases ${ }^{16,18}$.

Here, we explored the effects of glucose influx on vascular tone in humans and mice and found that blood glucose elevation induced vascular constriction in vivo. Mechanistically, glucose oxidation induced VSMC contraction through mitochondrial hyperpolarization-inactivated MLCP. These findings couple mitochondrial energetics with cell contraction in vascular smooth muscles, suggesting a new regulatory mechanism of vascular tone.

\section{Materials and methods Study population}

The study was approved by the Human Research Ethics Committee of Fourth Military Medical University. Eight adults (male, 25-35 years old) without high risks for development of or diagnosed cardiovascular diseases were recruited. Informed written consent was obtained from each participant before enrollment. Subject characteristics are shown in supplemental Table 1. Oral glucose challenge was performed after $12 \mathrm{~h}$ overnight fast. Two tests with a 5-day interval were carried out for each participant. After oral ingestion of glucose or mannitol $(1.0 \mathrm{~g} / \mathrm{kg}$ body weight, dissolved in $200 \mathrm{~mL}$ water) in less than $2 \mathrm{~min}$, blood glucose, BP, and ultrasonic imaging were measured at $0,30,60$, and $120 \mathrm{~min}$ after challenge. Glucose or mannitol was assigned randomly to each participant at the first test, and the other challenge was assigned in the second test. All parameters were detected with the participant in a supine position. BP was measured using standard mercury sphygmomanometers by experienced observers as described previously ${ }^{19}$. The average or the last of 2 consecutive readings was recorded. The investigator was blinded to the group allocation when detecting the parameters.

\section{Animal model}

Animal experiments were performed according to the National Institutes of Health Guidelines for the Use of Laboratory Animals, and were approved by Fourth Military Medical University Committee on Animal Care. C57 mice (male, 8 weeks) were purchased from the Animal Research Center of Fourth Military Medical University (China). Mice were randomly divided into different groups. The investigator was blinded to the group allocation when detecting the parameters. Mice were administered an intraperitoneal injection of glucose $(2 \mathrm{~g} / \mathrm{kg})$ for glucose challenge after $12 \mathrm{~h}$ overnight fast. Blood glucose, blood pressure, and ultrasonic imaging were determined at $0,15,30,60$, and $90 \mathrm{~min}$ after the glucose challenge. Mice were administered a hypodermic injection of insulin $(0.5 \mathrm{U} / \mathrm{kg})$ for insulin challenge after $12 \mathrm{~h}$ overnight fast. Blood glucose was detected at $0,30,60,90$, and $120 \mathrm{~min}$ post insulin challenge.

Mypt ${ }^{\mathrm{SMKO}}$ mice were generated, genotyped and raised as reported previously ${ }^{18,20}$. Briefly, the chimeric mice carrying floxed Mypt1 were crossed with SMA-Cre mice to ablate Mypt1 specifically in smooth muscle. Mice with 2 floxed Mypt1 alleles and SMA-Cre (Mypt $1^{\text {flox/flox }}$; SMA$\mathrm{Cre})$ were used as Mypt1 ${ }^{\text {SMKO }}$ mice. Male Mypt1 ${ }^{\text {SMKO }}$ mice and the corresponding WT mice ( $8 \mathrm{wk}$ ) were used for experiments. 


\section{Ultrasonography}

Ultrasonography was performed with the ACUSON Sequoia 512 ultrasound machine (Siemens, New Jersey, USA) for humans and the Vevo 2100 imaging system (VisualSonics, Toronto, Canada) for mice. Cardiac ultrasound was used to determine stroke volume and heart rate. Cardiac output was calculated by stroke volume $\times$ heart rate. Vascular ultrasound was used to determine the maximal diameter of a brachial artery or abdominal aorta during vascular relaxation. Blood glucose, brachial BP, and ultrasonic imaging were simultaneously detected in humans, while blood glucose, caudal BP, and ultrasonic imaging were parallelly detected in mice.

\section{Functional assessment of thoracic aorta}

Mice were sacrificed and the thoracic aortas were carefully excised and placed in ice-cold physiological saline solution (in $\mathrm{mM}: \mathrm{NaCl} 119, \mathrm{KCl} 4.6, \mathrm{MgCl}_{2}$ 1.2, $\mathrm{CaCl}_{2} 1.5, \mathrm{NaHCO}_{3} 15, \mathrm{NaH}_{2} \mathrm{PO}_{4} 1.2$, and glucose 5.0) as described previously ${ }^{21}$. The fat and connective tissue around the blood vessels were removed. The thoracic aorta segments $(1 \mathrm{~mm})$ were mounted in a temperaturecontrolled $\left(37^{\circ} \mathrm{C}\right)$ myograph system (DMT $610 \mathrm{M}$, Danish Myo Technology, Denmark) which was perfused with PSS continuously gassed with a mixture of $5 \% \mathrm{CO}_{2}$ and $95 \%$ $\mathrm{O}_{2}$ (pH 7.35-7.45). An optimal initial length was applied for 1 hour before experiments. Endothelium removal was achieved by mechanical abrasion. Thoracic aorta segments were precontracted with $0.1 \mu \mathrm{M}$ phenylephrine $(\mathrm{PE})$ to test the effects of different treatments (metabolites, ionophores, and others) on vascular tone. After precontraction, thoracic aortas were challenged with glucose $(30 \mathrm{mM})$, glucose $(10 \mathrm{mM})+$ insulin $(10 \mathrm{nM})$, FCCP $(1 \mu \mathrm{M})$, nigericin $(1 \mu \mathrm{M})$, valinomycin $(1 \mu \mathrm{M})$, monesin $(1 \mu \mathrm{M}), \mathrm{NH}_{4}{ }^{+}(5 \mathrm{mM}), \mathrm{Ch}_{3} \mathrm{COO}^{-}(5 \mathrm{mM})$, thapsigargin (TG) $(5 \mu \mathrm{M})$, or BAPTA-AM $(10 \mu \mathrm{M})$, unless noted otherwise.

For measurement of vascular function of vasodilation, aorta segments were precontracted with PE $(10 \mu \mathrm{M})$ before treatments with acetylcholine (ACh) and sodium nitroprusside (SNP). Vasodilation evoked by cumulative ACh $\left(10^{-9}\right.$ to $\left.10^{-5}\right)$ or SNP $\left(10^{-10}\right.$ to $\left.10^{-5} \mathrm{M}\right)$ was determined and the results were expressed as the percentage of PE-induced contractile force. For measurement of vasoconstriction, aorta segments were not precontracted with PE and cumulative PE $\left(10^{-9}\right.$ to $\left.10^{-5} \mathrm{M}\right)$ and angiotensin II $\left(10^{-11}\right.$ to $\left.10^{-7} \mathrm{M}\right)$ were used to evoke vasoconstriction.

\section{VSMCs isolation and culture}

Primary VSMCs were isolated from mouse thoracic aortas and cultured in DMEM as described previously ${ }^{22}$. Briefly, WT or Mypt $1^{\text {SMKO }}$ mice were anesthetized by sodium pentobarbital (200 mg/kg, i.p.). The thoracic aorta was dissected, and the adhering periadventitial tissue and endothelium were removed. After removing the adventitial layer with Collagenase I solution (Sigma, $1 \mathrm{mg} / \mathrm{ml}$ ) for $10 \mathrm{~min}$ at $37^{\circ} \mathrm{C}$, the medial layer was minced into small pieces for second digestion with Collagenase I for $2 \mathrm{~h}$. Isolated VSMCs were cultured in DMEM containing $5 \mathrm{mM}$ glucose, $10 \% \mathrm{FBS}$, and $1 \%$ penicillin/streptomycinglutamine. VSMC purity was assessed by staining of smooth muscle-specific $\alpha$-actin antibody (Santa). VSMCs between passages 2 (P2) to P5 were used. After serum starvation for $24 \mathrm{~h}$, VSMCs were challenged with glucose $(30 \mathrm{mM})$, glucose $(10 \mathrm{mM})+$ insulin $(10 \mathrm{nM})$, FCCP $(1 \mu \mathrm{M})$, nigericin $(1 \mu \mathrm{M}), \mathrm{NH}_{4}{ }^{+}(5 \mathrm{mM})$ or $\mathrm{Ch}_{3} \mathrm{COO}^{-}$ $(5 \mathrm{mM})$ for $10 \mathrm{~min}$, unless noted otherwise. VSMCs were pretreated with $\alpha$-cyano-4-hydroxycinnamic acid ( $\alpha$-CCA, $200 \mu \mathrm{M})$, FCCP $(1 \mu \mathrm{M})$, nigericin $(1 \mu \mathrm{M})$, TG $(5 \mu \mathrm{M})$, BAPTA-AM $(10 \mu \mathrm{M})$, Y27632 $(1 \mu \mathrm{M})$, or Fasudil $(10 \mu \mathrm{M})$ at $30 \mathrm{~min}$ before metabolic challenges.

\section{Small interfering RNA (siRNA) transfection}

For gene silencing assay, siRNAs for RhoA and MYPT1 were designed and purchased from GenePharma (Shanghai, China). The sequences of the oligos are shown in supplemental Table 2. VSMCs were transfected with siRNA or negative control by Lipofectamine ${ }^{\mathrm{TM}} 2000$ (Invitrogen) following the manufacturer's instructions. The efficiency of gene knockdown was detected by western blot at $60 \mathrm{~h}$ after siRNA transfection.

\section{Confocal imaging}

An inverted confocal microscope (Zeiss LSM 800, 40×, 1.3 NA oil-immersion objective) was used for imaging. Cells or blood vessels were incubated with Tyrode's solution consisting of (in $\mathrm{mM}: \mathrm{NaCl} 137, \mathrm{KCl} 5.4, \mathrm{MgCl}_{2}$ 1.2, $\mathrm{NaH}_{2} \mathrm{PO}_{4}$ 1.2, $1.0 \mathrm{CaCl}_{2}$, glucose 5.0, and HEPES 20, $\mathrm{pH}$ 7.35-7.45). For measurement of $\Delta \Psi \mathrm{m}, \mathrm{JC}-1(50 \mu \mathrm{M})$ or TMRM $(20 \mathrm{nM})$ was loaded at $37^{\circ} \mathrm{C}$ for $20 \mathrm{~min}$ followed by 3 times washing with Tyrode's solution. To obtain JC-1 signals, images were captured by tandem excitation of scan-lines at $488 \mathrm{~nm}$ and $573 \mathrm{~nm}$, and emission collection at $500-570 \mathrm{~nm}$ (monomer) and 600-670 $\mathrm{nm}$ (aggregate). The fluorescence ratio (monomer/aggregate) indicates the changes of $\Delta \Psi \mathrm{m}$. To obtain TMRM signals, images were captured by excitation at $573 \mathrm{~nm}$ and emission collection at $600-650 \mathrm{~nm}$. All experiments were performed at room temperature $\left(22-24{ }^{\circ} \mathrm{C}\right)$. For measurement of $\Delta \Psi \mathrm{m}$ within the smooth muscle of intact thoracic aorta, the arteries were maintained at physiological pressure as described previously ${ }^{14}$.

\section{Western blot}

Proteins were extracted from either blood vessels or VSMCs. The levels of protein expression or phosphorylation were measured using Western blot as described 
previously ${ }^{23}$. The immunoblots were probed with anti-pMLC (Ser20), anti-MLC, anti-p-MYPT1 (Thr853), antiMYPT1, anti-ROCK1, anti-ROCK2, anti-p-CPI-17 (Thr38), anti-CPI-17, anti-RhoA, and anti- $\beta$-actin antibodies (Abcam/Cell Signaling Technology) overnight at $4{ }^{\circ} \mathrm{C}$ followed by incubation with the corresponding secondary antibodies at room temperature for $1 \mathrm{~h}$. RhoA activation was measured with the Rho Activation Assay Kit from Cell Biolabs according to the manufacturer's instructions (STA-403-A).

\section{Circulating metabolite clearance}

The mice were injected with a bolus of 2-NBDG $(10 \mu \mathrm{g} /$ $\mathrm{g})$, a fluorescent-labeled glucose analogy, or indocyanine green (ICG) $(10 \mu \mathrm{g} / \mathrm{g})$, a low-toxic fluorescent that is selectively taken up by hepatocytes, via caudal vein $(0.2 \mathrm{~mL})$ after $12 \mathrm{~h}$ overnight fast. Meanwhile, glucose $(2 \mathrm{~g} / \mathrm{kg})$ or saline was intraperitoneal injected. Blood samples were taken at $5,30,60,90$, and $120 \mathrm{~min}$ after injection. The 2-NBDG and ICG fluorescence in serum was determined spectrophotometrically at 488 and $800 \mathrm{~nm}$, respectively.

\section{Statistical analysis}

The sample size for the human study was estimated using a sample size calculator (www.calculator.net).
Sample sizes for other experiments were chosen based on studies with similar experimental design and on the known variability of the assay. All values are presented as mean \pm SEM. Data were compared with one-way ANOVA or two-way ANOVA, with all ANOVA tests followed by a paired (for human data) or unpaired (for other data) twotailed t-test, as appropriate. Bonferroni's correction for multiple comparisons was used. Linear relationships between variables were tested by Pearson's correlation coefficient. Normal distribution of data was analyzed by Kolmogorov-Smirnov normality test. In all statistical comparisons, $p$ value of less than 0.05 was considered to be statistically significant. No samples/animals were excluded, except excluding some immunofluorescent data prior to analysis due to techinical issues (out of focus, auto-fluorescent debris, etc).

\section{Results}

Glucose challenge induced vascular constriction in vivo

To examine the effects of glucose influx on vascular tone, a total of 8 healthy adults (male, 25-35 years old) were recruited. Oral glucose challenge $(1.0 \mathrm{~g} / \mathrm{kg}$ body weight) resulted in a transient increase in blood glucose, reaching its peak level at $30 \mathrm{~min}$ and falling to euglycemia at $2 \mathrm{~h}$ post-challenge (Fig. 1A). Simultaneous measurement of BP and cardiac function showed that systolic BP
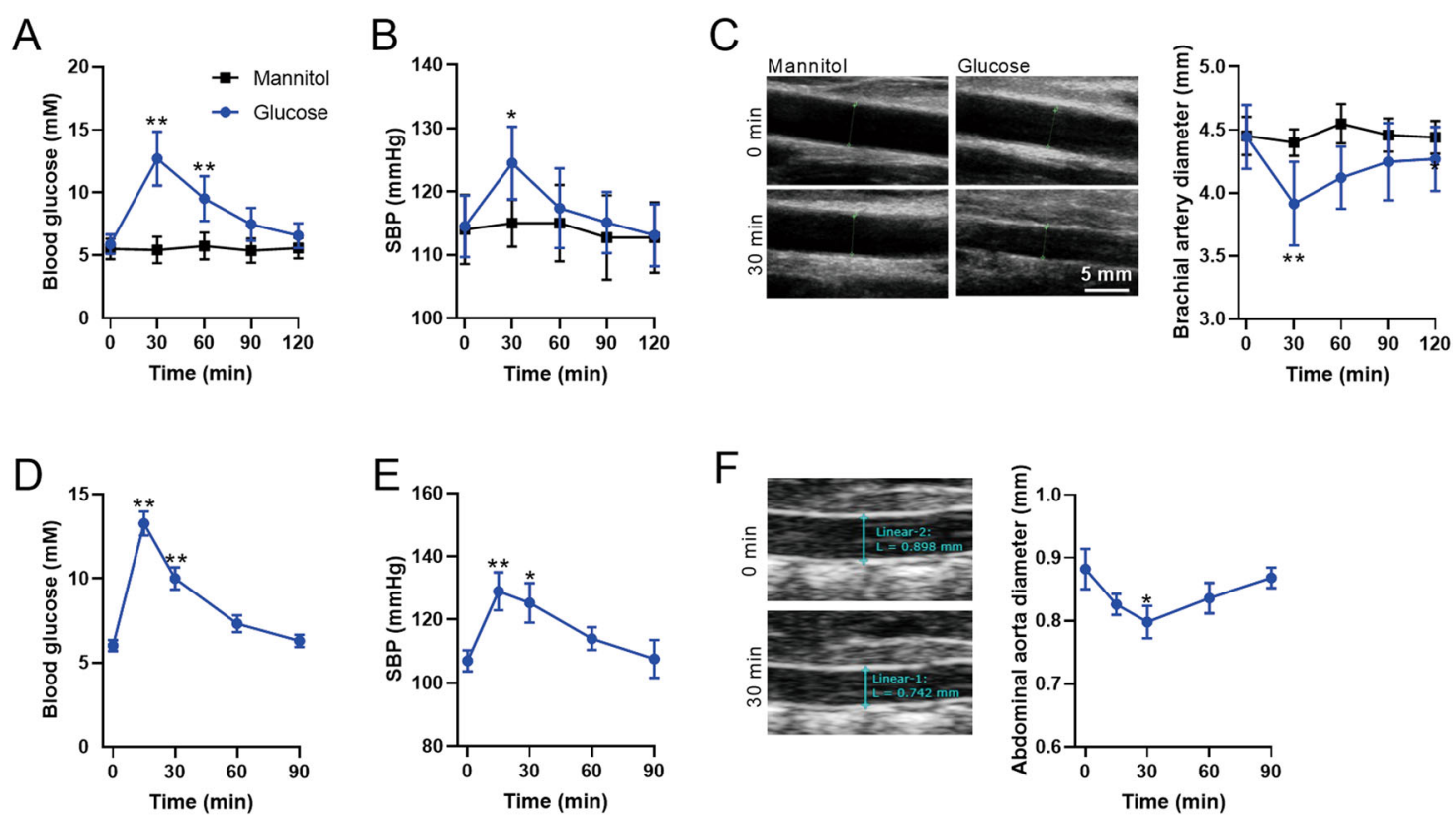

Fig. 1 Glucose challenge induced vascular constriction in humans and mice. A-F Blood glucose (A), systolic blood pressure (SBP) (B) and the maximal brachial artery diameter $(\mathbf{C})$ in response to oral glucose challenge in humans. Representative images of the maximal diameter of brachial artery in response to glucose challenge are shown in left, and the quantified results are shown in right $(\mathbf{C}) . n=8$ subjects. D-F Blood glucose (D), SBP $(\mathbf{E})$, and the maximal abdominal aorta diameter $(\mathbf{F})$ in response to intraperitoneal glucose challenge in mice. Representative images of the maximal abdominal aorta in response to glucose challenge are shown in left, and the quantified results are shown in right $(\mathbf{F}) . n=8$ mice. Error bars represent SEM. ${ }^{*} P<0.05 .{ }^{* *} P<0.01$. 


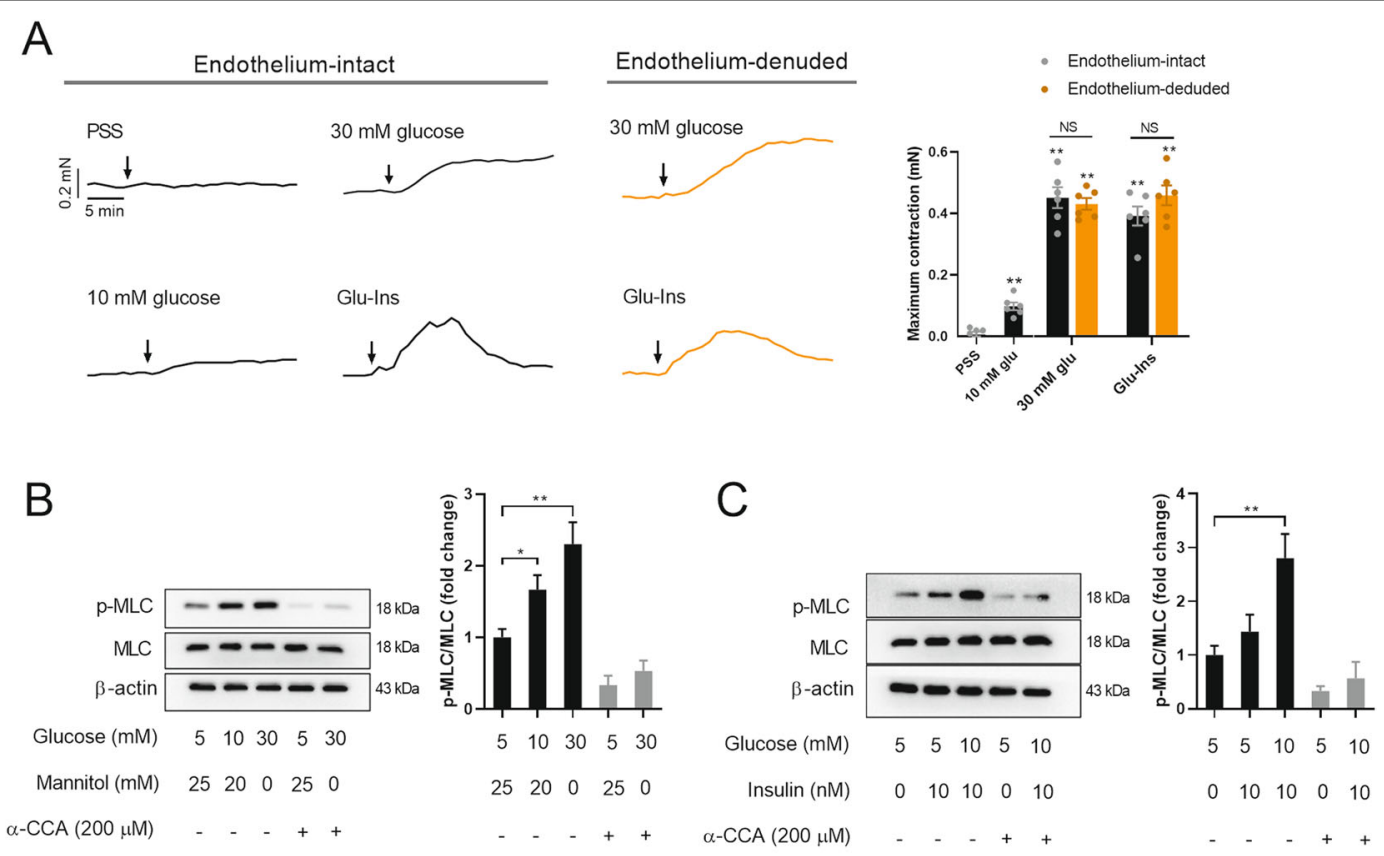

D

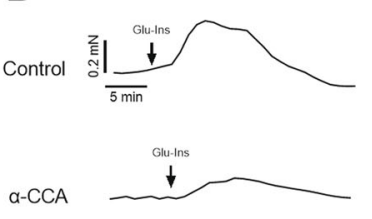

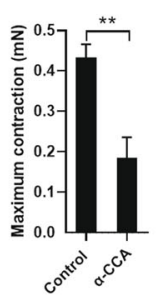

E

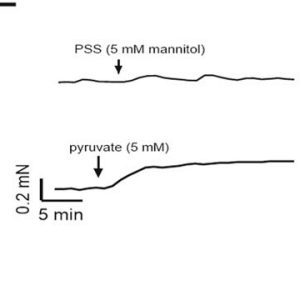

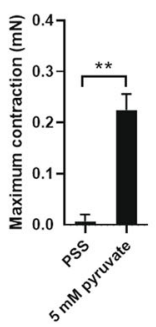

Fig. 2 Glucose-induced vascular constriction is dependent on mitochondrial oxidation in VSMCs. A Glucose (10, $30 \mathrm{mM})$ and Glu-Ins (10 mM glucose $+10 \mathrm{nM}$ insulin) induced vascular constriction in isolated thoracic aortas with intact endothelium or with denuded endothelium. The aortas were pretreated with PE. Typical tension curves of thoracic aortas were shown in the left, and the quantified results were shown in right. Osmotic pressure was adjusted with mannitol. ${ }^{*}$, vs. saline. $n=6$. B, C Glucose (B) or Glu-Ins (C) induced MLC phosphorylation, which was blocked by a-CCA in cultured VSMCs. $n=5$. $\mathbf{D}$ a-CCA pretreatment blocked the effects of Glu-Ins on induction of vascular constriction in isolated thoracic aortas. The aortas were pretreated with PE. $n=6$. E Pyruvate induced vascular constriction in isolated thoracic aortas. The aortas were pretreated with PE. $n=6$. Error bars represent SEM. ${ }^{*} P<0.05$. $^{* *} P<0.01$.

was increased by $8.7 \%$ at $30 \mathrm{~min}$ post-challenge (Fig. 1B), while diastolic BP and cardiac output displayed no significant differences (supplemental Fig. 1), suggesting that an increase in systemic vascular resistance may underlie the evaluation of systolic BP. In fact, a decrease in the maximal diameter of the brachial artery was observed (decreased by $12.0 \%$ ) at $30 \mathrm{~min}$ post challenge (Fig. 1C). There was a negative linear relationship between blood glucose and the maximal brachial artery diameter in subjects with glucose challenge (supplemental Fig. 1). Similarly, intraperitoneal glucose challenge $(2 \mathrm{~g} / \mathrm{kg}$ body weight) resulted in transient increases in blood glucose and systolic BP in mice (Fig. 1D, E). In addition, parallelly measurement of the maximal abdominal aorta diameter revealed that abdominal aorta diameter was decreased at
$30 \mathrm{~min}$ post-challenge in mice (Fig. 1F). These results suggested that glucose challenge increases vascular constriction and systolic BP in both humans and mice.

\section{Glucose-induced vascular constriction is dependent on mitochondrial oxidation in VSMCs}

To test whether glucose induces vascular constriction directly, the effect of glucose on vascular constriction was determined ex vivo. As shown in Fig. 2A, glucose (10 and $30 \mathrm{mM}$ ) induced thoracic aorta constriction in a dosedependent manner. To mimic the glucose challenge in vivo, a mixture of glucose and insulin $(10 \mathrm{mM}$ glucose and $10 \mathrm{nM}$ insulin, Glu-Ins) was used. Glu-Ins induced vascular constriction to an extent similar to that of $30 \mathrm{mM}$ glucose (Fig. 2A). However, Glu-Ins-induced vascular 
constriction was reversible, reaching its peak level at $12.2 \pm 2.3 \mathrm{~min}$ and falling to baseline at $25.2 \pm 4.5 \mathrm{~min}$ (Fig. 2A), probably due to a transient response of insulin (supplemental Fig. 2). On the contrary, glucose-induced vascular constriction was relatively stable and lasted at least for $60 \mathrm{~min}$. These results suggested that glucose metabolism directly stimulates vascular constriction.

Endothelium removal showed no significant effect on glucose- or Glu-Ins-induced vascular constriction ex vivo, suggesting that glucose-induced vascular constriction is VSMC-dependent (Fig. 2A). As expected, glucose and GluIns increased MLC phosphorylation in cultured VSMCs (Fig. 2B, C). Interestingly, both glucose- and Glu-Insinduced MLC phosphorylation were blocked by $\alpha$-CCA $(200 \mu \mathrm{M})$, an inhibitor of the mitochondrial pyruvate transporter, suggesting that glucose oxidation is necessary in glucose-induced VSMC contraction (Fig. 2B, C). $\alpha$-CCA pretreatment $(200 \mu \mathrm{M})$ also blocked the effects of Glu-Ins on induction of vascular constriction in isolated thoracic aortas (Fig. 2D). In fact, glucose increased mitochondrial oxidation as evidenced by increased respiration (supplemental Fig. 3). Furthermore, pyruvate, one of the end products of glycolysis, induced vascular constriction in isolated thoracic aortas (Fig. 2E). These results suggested that glucose-induced vascular constriction is dependent on mitochondrial oxidation in VSMCs.

\section{Mitochondrial membrane potential positively regulates VSMC contraction}

In another set of experiments, we aimed to investigate which component in mitochondrial oxidation is involved in the regulation of VSMC contraction using four ionophores. FCCP, an electrical $\mathrm{H}^{+}$ionophore, dissipating proton gradient $(\Delta \mathrm{pH})$ and eliminating $\Delta \Psi \mathrm{m}$ and mitochondrial ATP production ${ }^{24}$. Valinomycin, an electrical $\mathrm{K}^{+}$ionophore, depolarizes $\Delta \Psi \mathrm{m}$ and eliminates mitochondrial ATP formation ${ }^{24}$. Nigericin, an electroneutral $\mathrm{H}^{+} / \mathrm{K}^{+}$ionophore, eliminates $\Delta \mathrm{pH}$ and mitochondrial ATP production and results in a compensating increase in $\Delta \Psi \mathrm{m}^{24}$. Monesin, an electroneutral $\mathrm{H}^{+} / \mathrm{Na}^{+}$ionophore, exerts the similar effects as that for nigericin. The results showed that FCCP and valinomycin disrupted vascular constriction to baseline, while nigericin and monensin increased vascular constriction in both endotheliumintact and endothelium-denuded aortas (Fig. 3A). These results indicated that $\Delta \Psi \mathrm{m}$ is a potential regulator of VSMC contraction, while mitochondrial ATP formation is not necessary in the induction of VSMC contraction. The effects of these ionophores on $\Delta \Psi \mathrm{m}$ in VSMCs were shown in Fig. 3B. To exclude non-specific effects of these ionophores on $\Delta \Psi \mathrm{m}$, oligomycin which blocks mitochondrial ATP production and increases $\Delta \Psi \mathrm{m}$ was used. Oligomycin also increased vascular constriction in isolated thoracic aortas (Fig. 3C).
Although mitochondrial ATP formation is not necessary for mitochondrial hyperpolarization-induced vascular constriction, ATP from glycolysis is necessary as evidenced by that all these ionophores disrupted vascular constriction when pyruvate $(5 \mathrm{mM})$ was supplied as the only metabolite in the incubation medium (Fig. 3A). To extend our findings, $\mathrm{CH}_{3} \mathrm{COO}^{-}(5 \mathrm{mM})$ and $\mathrm{NH}_{4}{ }^{+}$ $(5 \mathrm{mM})$, classic tools in the intervention of $\Delta \Psi \mathrm{m}$, were employed. $\mathrm{CH}_{3} \mathrm{COO}^{-}$depolarized $\Delta \Psi \mathrm{m}$ and $\mathrm{NH}_{4}^{-}$ hyperpolarized $\Delta \Psi \mathrm{m}$. As a result, $\mathrm{CH}_{3} \mathrm{COO}^{-}$induced vascular relaxation and $\mathrm{NH}_{4}{ }^{+}$promoted vascular constriction in a dose-dependent manner in isolated aortas (Fig. 3D). It should be noted that $\mathrm{CH}_{3} \mathrm{COO}^{-}$and $\mathrm{NH}_{4}{ }^{+}$ also affect mitochondrial $\mathrm{pH}$. These results reinforced the notion that $\Delta \Psi \mathrm{m}$ positively regulates VSMC contraction.

\section{Mitochondrial hyperpolarization contributes to glucose- induced VSMC contraction}

To test whether glucose-induced vascular constriction is dependent on mitochondrial hyperpolarization, FCCP and nigericin were used to clamp $\Delta \Psi \mathrm{m}$ in isolated aortas and cultured VSMCs. Glu-Ins induced an increase in $\Delta \Psi \mathrm{m}$ in VSMCs of isolated aortas (Fig. 3E), and preincubation of isolated aortas with either FCCP or nigericin inhibited the Glu-Ins-induced mitochondrial hyperpolarization (Fig. 3E). As a result, Glu-Ins-induced vascular constriction was inhibited in isolated aortas preincubated with FCCP or nigericin (Fig. 3F). In addition, preincubations of cultured VSMCs with FCCP or nigericin blocked the mitochondrial hyperpolarization, as well as MLC phosphorylation, induced by glucose $(30 \mathrm{mM})$ (supplemental Fig. 4). These results suggested that mitochondrial hyperpolarization contributes to glucose-induced VSMC contraction.

\section{Glucose induces VSMC contraction through inhibition of MLCP}

To explore the underlying mechanism, we initially tested whether $\mathrm{Ca}^{2+}$ signaling is involved in glucose-induced vascular constriction. As shown in Fig. 4A, glucose and Glu-Ins showed little effects on intracellular $\mathrm{Ca}^{2+}$ as detected by fluo-4 in cultured VSMCs. In addition, FCCP, $\mathrm{NH}_{4}{ }^{+}$and $\mathrm{Ch}_{3} \mathrm{COO}^{-}$increased intracellular $\mathrm{Ca}^{2+}$ levels, while nigericin decreased intracellular $\mathrm{Ca}^{2+}$ levels in cultured VSMCs, indicating $\triangle \Psi \mathrm{m}$-regulated VSMC contraction independently of $\mathrm{Ca}^{2+}$ signaling (Fig. 4B). Furthermore, Glu-Ins or glucose still promoted VSMC contraction in isolated aortas and cultured VSMCs even when the aortas or VSMCs were pretreated with TG, an endoplasmic reticular $\mathrm{Ca}^{2+}$-ATPase inhibitor, or BAPTA-AM, a cell-permeable cytosolic calcium chelator (Fig. 4C, D). These results suggested that glucose-induced VSMC contraction is not likely to be mediated by intracellular $\mathrm{Ca}^{2+}$ signaling.

In addition to the $\mathrm{Ca}^{2+}$-dependent activation of MLCK, MLCP is the other component in the regulation of VSMC 
A

Ionophore Endothelium-intact Endothelium-denuded $\frac{\text { Pyruvate as substrate }}{\text { Endothelium-intact }}$

FCCP
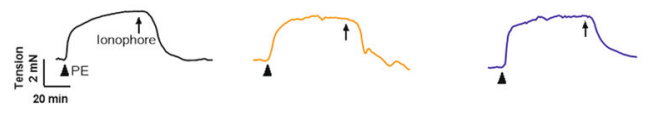

Valinomycin
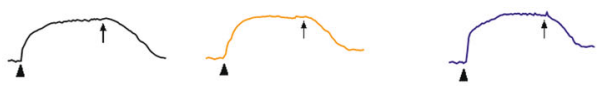

Nigericin
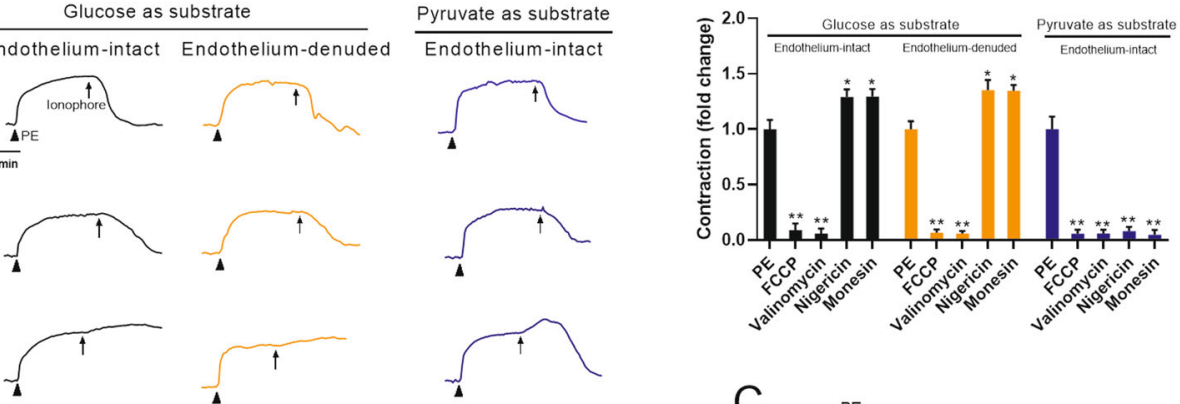

Monesin
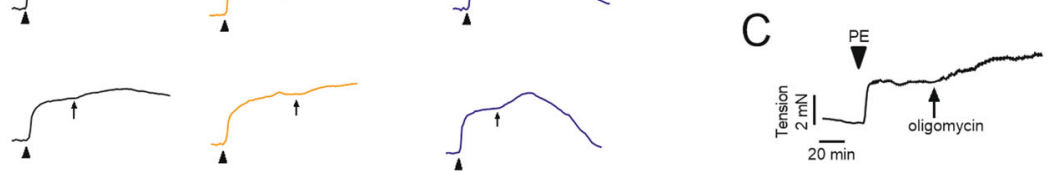

B $\quad F C C P$
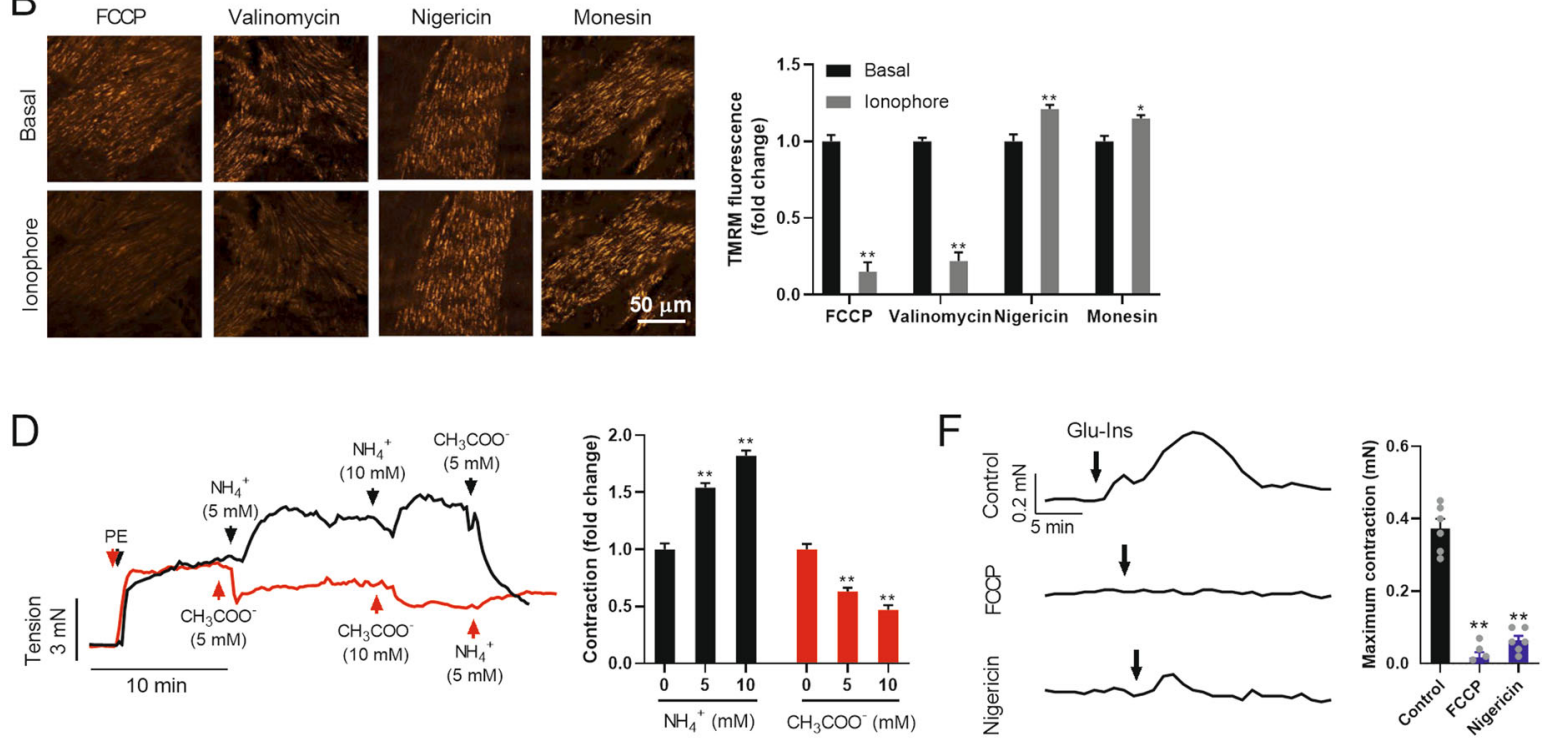

E $\frac{\text { Vehicle }}{\text { Basal Glu-Ins }}$
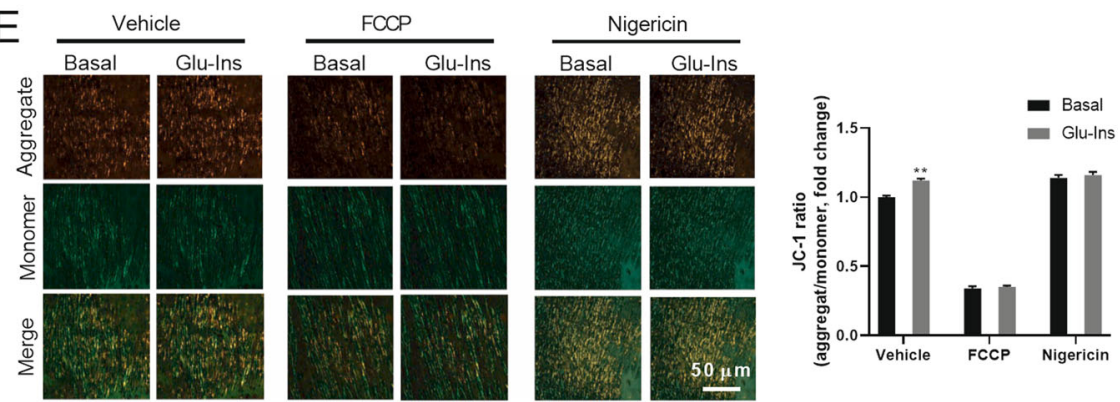

Fig. $3 \Delta \Psi_{m}$ positively regulates VSMC contraction. A The effects of four ionophores on vascular constriction in isolated thoracic aortas with intact endothelium or with denuded endothelium. The aortas were pretreated with PE. Glucose $(5 \mathrm{mM})$ or pyruvate $(5 \mathrm{mM})$ was supplied as the only metabolite available in the incubation medium. Typical traces of vascular tension were shown in the left, and the quantified results were shown in right. The arrow indicates the time point for treatment with ionophore. $n=6$. B The effects of four ionophores on $\Delta \Psi_{\mathrm{m}}$ in VSMCs of isolated aortas. Typical images of $\Delta \Psi_{\mathrm{m}}$ in VSMCs in blood vessels were shown in the left, and the quantified results were shown in right. $\Delta \Psi_{\mathrm{m}}$ was detected by TMRM. $n=6$. C Oligomycin induced vascular constriction in isolated thoracic aortas. D The effects of $\mathrm{NH}^{4+}$ and $\mathrm{CH}_{3} \mathrm{COO}^{-}$on vascular tone in isolated aortas. Typical traces of vascular tension were shown in the left, and the quantified results were shown in right. $n=6$. $\mathbf{E}$ Glu-Ins-induced mitochondrial hyperpolarization was blocked by preincubation of isolated aortas with FCCP or nigericin. $\Delta \Psi_{m}$ was detected by JC-1. Typical images of $\Delta \Psi_{m}$ in VSMCs in blood vessels were shown in the left, and the quantified results were shown in right. $\mathbf{F}$ Preincubation of isolated aortas with FCCP or nigericin inhibited Glu-Ins-induced vascular constriction. Typical traces of vascular tension were shown in the left, and the quantified results were shown in right. $n=6$. Error bars represent $\operatorname{SEM} .{ }^{*} P<0.05$. ${ }^{* *} P<0.01$. 
A

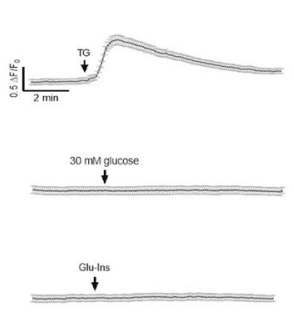

D
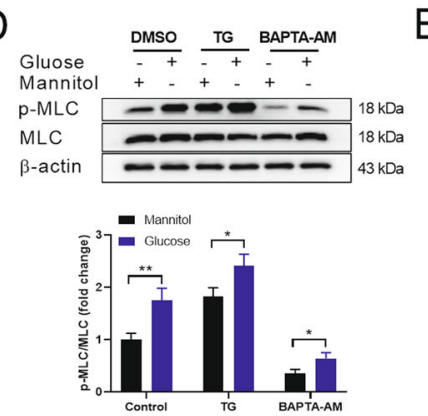

B

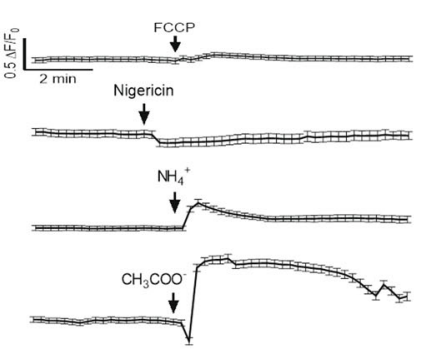

E

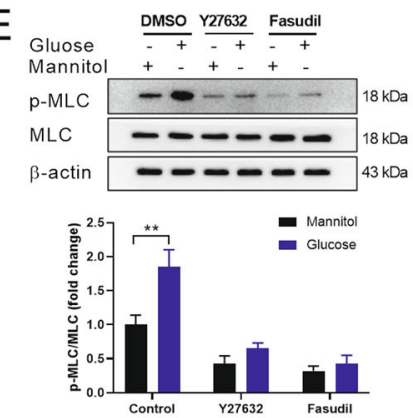

C

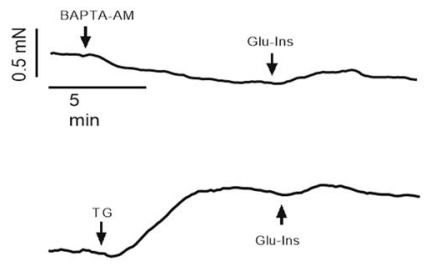

$\mathrm{F}$

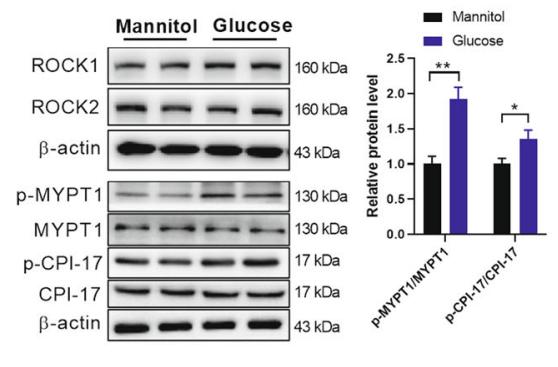

G
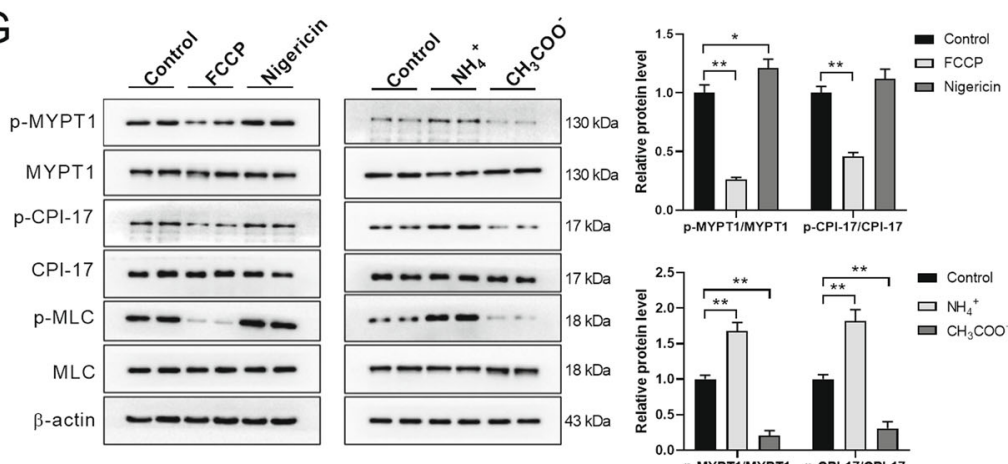

$\mathrm{H}$

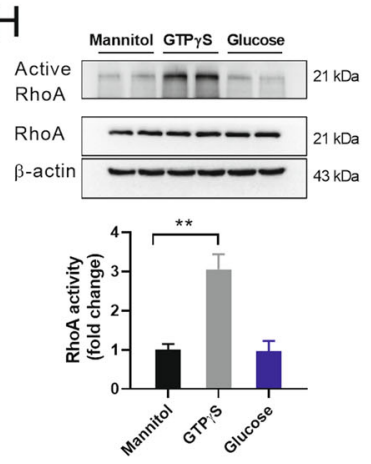

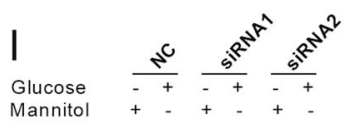

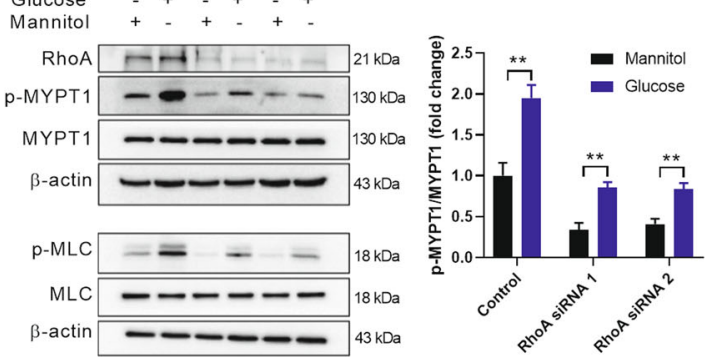

$J$
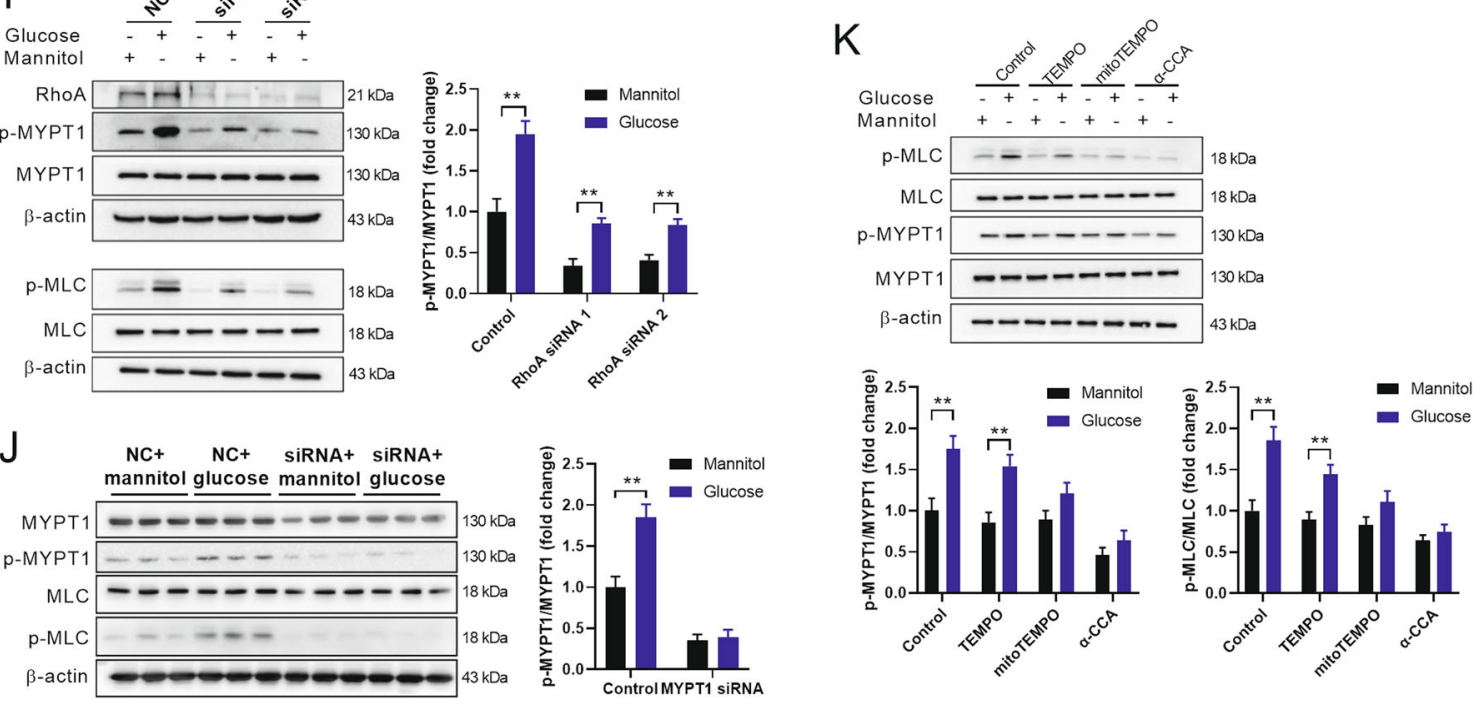

Fig. 4 (See legend on next page.) 
(see figure on previous page)

Fig. 4 Glucose induces VSMC contraction through inhibition of MLCP. A The effects of glucose $(30 \mathrm{mM})$ and Glu-Ins on intracellular Ca ${ }^{2+}$ in VSMCs. $n=5$. B The effects of FCCP, nigericin, $\mathrm{CH}_{3} \mathrm{COO}^{-}$and $\mathrm{NH}_{4}{ }^{+}$on intracellular $\mathrm{Ca}^{2+}$ in VSMCs. $n=5$. C The effects of Glu-Ins on vascular constriction in isolated aortas preincubated with either thapsigargin (TG) or BAPTA-AM. $n=5$. D The effects of glucose (30 mM) on MLC phosphorylation in cultured VSMCs preincubated with either TG or BAPTA-AM. $n=5$. $\mathbf{E}$ The effects of glucose ( $30 \mathrm{mM})$ on MLC phosphorylation in cultured VSMCs preincubated with either Y27632 or Fasudil. $n=5$. F Glucose (30 mM) increased phosphorylation of MYPT1 and CPI-17 in cultured VSMCs. $n=5$. G Nigericin and $\mathrm{NH}_{4}{ }^{+}$increased MYPT1 phosphorylation and FCCP and $\mathrm{CH}_{3} \mathrm{CHOO}^{-}$decreased MYPT1 phosphorylation in cultured VSMCs. $n=5$. $\mathbf{H}$ Glucose $(30 \mathrm{mM})$ showed little effects on both RhoA expression and activity in cultured VSMCs. $n=4$. I Knockdown of RhoA did not block the effects of glucose $(30 \mathrm{mM})$ on phosphorylation of MLC and MYPT1 in cultured VSMCs. $n=5$. J Knockdown of MYPT1 impaired the effects of glucose $(30 \mathrm{mM})$ on MLC phosphorylation in cultured VSMCs. $n=5$. $\mathbf{K}$ The effects of TEMPO, mitoTEMPO, and a-CCA pretreatments on glucose influx-induced phosphorylation of MYPT1 and MLC in VSMCs. $n=5$. Error bars represent SEM. ${ }^{*} P<0.05 .{ }^{* *} P<0.01$.

contraction. To evaluate this possibility, we first explored the effects of 2 distinct non-isoform-selective ROCK inhibitors, Y27632 and Fasudil ${ }^{25}$. Both Y27632 and Fasudil inhibited glucose-induced MLC phosphorylation in VSMCs (Fig. 4E). In addition, glucose increased the phosphorylation levels of both MYPT1 and CPI-17 by $92 \%$ and 35\%, respectively (Fig. 4F), suggesting that glucose inhibits MLCP activity. Consistently, nigericin and $\mathrm{NH}_{4}{ }^{+}$increased MYPT1 phosphorylation and FCCP and $\mathrm{CH}_{3} \mathrm{CHOO}^{-}$decreased MYPT1 phosphorylation (Fig. 4G). However, glucose showed little effect on the expression and activity of RhoA, as well as other molecules of Rho family (Fig. 4H and supplemental Fig. 5). Knockdown of RhoA did not block the effects of glucose on phosphorylation of MLC and MYPT1 (Fig. 4I), suggesting that glucose-induced VSMC contraction is not mediated by RhoA. Although the upstream regulator of MLCP has not been defined, knockdown of MYPT1 impaired the effects of glucose on VSMC contraction (Fig. 4J), suggesting that MYPT1 plays a critical role in regulation of smooth muscle tone by glucose. Since mitochondrial hyperpolarization facilities ROS production and ROS plays an important role in regulating various cellular signals, the role of ROS in glucose influx-induced MYPT1 phosphorylation has been tested. Pretreatment of VSMCs with TEMPO $(1 \mu \mathrm{M})$, a ROS scavenger, showed no significant effects on glucose-induced MYPT1 phosphorylation, while pretreatment of VSMCs with mitoTEMPO $(1 \mu \mathrm{M})$, a mitochondrial ROS scavenger, abolished the effects of glucose-induced MYPT1 phosphorylation (Fig. 4K), suggesting that mitochondrial hyperpolarization was not likely to phosphorylate MYPT1 through increasing cytosolic ROS. In addition, $\alpha$-CCA pretreatment $(200 \mu \mathrm{M})$ also abolished the effects of glucose-induced MYPT1 phosphorylation. These results suggested that glucose-induced VSMC contraction is mediated by the inactivation of MLCP.

\section{Smooth muscle-specific KO of MYPT1 impaired the effects of glucose on VSMC contraction}

To further test the role of MYPT1 in glucose-induced VSMC contraction, a mice model of smooth muscle-specific deletion of MYPT1 (Mypt1 ${ }^{\text {SMKO }}$ ) were used. Western blots confirmed no detectable MYPT1 expression in thoracic aortas, and MYPT1 KO showed little effect on expressions of other proteins in MLCP signaling (Fig. 5A). VSMC $\Delta \Psi \mathrm{m}$ in response to glucose, Glu-Ins, $\mathrm{NH}_{4}^{+}$, and $\mathrm{CH}_{3} \mathrm{COO}^{-}$was initially detected, the results displayed no differences between isolated aortas from WT and $\mathrm{Mypt}^{\mathrm{SMKO}}$ mice (Fig. 5B). However, the vascular constriction was impaired in response to glucose, Glu-Ins and $\mathrm{NH}_{4}{ }^{+}$, and vascular relaxation was attenuated in response to $\mathrm{CH}_{3} \mathrm{COO}^{-}$, in isolated aortas from Mypt $1^{\mathrm{SMKO}}$ mice compared with that from WT mice (Fig. 5C, D). Similarly, MYPT1 KO displayed little effects on $\Delta \Psi \mathrm{m}$ in response to glucose (Fig. $5 \mathrm{E}$ ), but decreased MLC phosphorylation in response to glucose in cultured VSMCs (Fig. 5F). These results reinforced the notion that glucose-induced VSMC contraction is mediated by phosphorylation of MYPT1.

\section{Mypt $1^{\mathrm{SMKO}}$ mice displayed glucose intolerance and impaired circulating metabolite clearance}

Mypt $1^{\mathrm{SMKO}}$ mice displayed normal fasting blood glucose and serum insulin (Fig. 6A, B), but increased systolic and diastolic BP (Fig. 6C), indicating that MYPT1 KO resulted in vascular dysfunction. MYPT1 KO enhanced vascular contraction of isolated aortas in response to $\mathrm{PE}$ and Angiotensin II (Fig. 6D, E), and inhibited vascular relaxation in response to SNP and ACh (Fig. 6F, G). Although the blood glucose was increased higher in response to glucose challenge (Fig. $6 \mathrm{H}$ ), systolic BP and vascular constriction showed little changes in Mypt $1^{\text {SMKO }}$ mice (Fig. 6I, J). In addition, insulin tolerance was not changed in response to insulin challenge in $\mathrm{Mypt}^{\mathrm{SMKO}}$ mice (Fig. 6K), suggesting that glucose-induced vascular contraction may be involved in the regulation of metabolite clearance. To test the hypothesis, we used 2-NBDG and ICG to trace the metabolite clearance in circulation. As shown in Fig. 6L, M, both the clearance rates of 2NBDG and ICG were enhanced by glucose challenge in WT mice, while the effects of glucose challenge on promoting the clearance rates of 2-NBDG and ICG were attenuated in $M y p t 1^{\text {SMKO }}$ mice. These results suggested that glucose-induced vascular constriction contributes to circulating metabolite clearance in mice. 


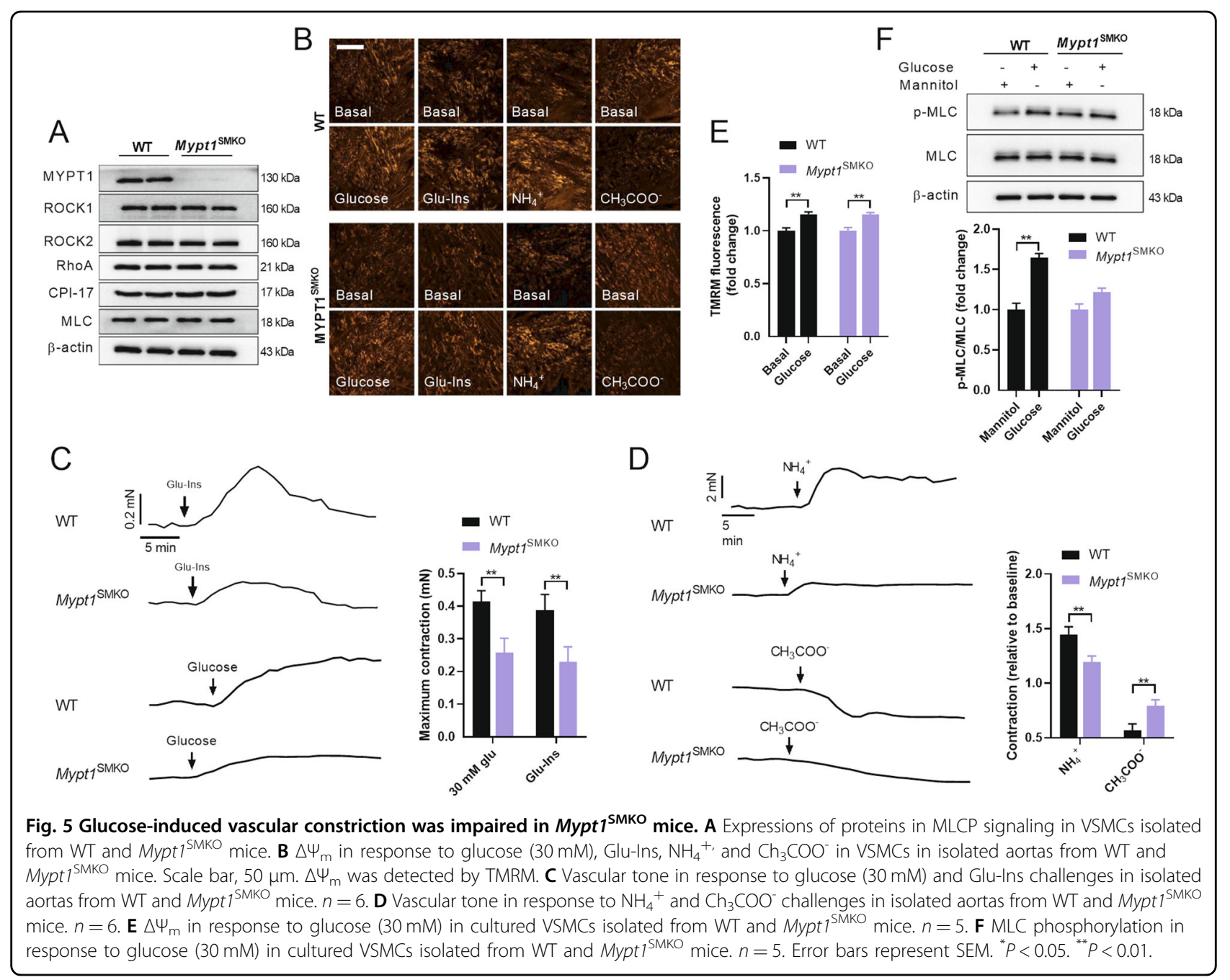

\section{Discussion}

Long-term exposure of the vasculature to metabolic disturbances leaves a persistent imprint on VSMCs, which potentially underlies macrovascular complications in metabolic disorders. However, the physiological effects of metabolic influx on vascular tone regulation remain largely unknown. Here, we found that glucose influx induces vascular constriction through mitochondrial hyperpolarization in VSMCs. Mechanistically, $\underline{\Psi m}$ positively phosphorylates MYPT1 which increases MLC phosphorylation through inhibition of MLCP (Fig. 6N). These findings represent a novel mechanism underlying vascular constriction.

Although BP maintains constant as a result of intrinsic cardiovascular regulatory mechanisms, it is characterized by marked fluctuations that have important significance in both physiological and pathological processes ${ }^{26,27}$. For example, a temporary spike in BP in response to stress facilitates blood perfusion to important organs to increase energy supply. Here we showed that glucose influx induces temporary spikes in vascular constriction and BP, which facilitate organ perfusion and metabolite clearance in circulation. A recent study also showed that glucose influx increased vascular tone in cerebral arteries ${ }^{28}$. In addition, we found that glucose challenge promoted clearance of glucose, 2-NBDG, and ICG in circulation, and these responses were attenuated in Mypt $1^{\mathrm{SMKO}}$ mice, indicating that blood glucose fluctuation plays a physiological role in the regulation of circulating metabolite homeostasis. Transient increase in blood glucose is observed in various physiological conditions, such as feeding behavior and acute stress. A temporary spike in $\mathrm{BP}$ in response to acute stress has been well-documented, while the observations of postprandial BP are not consistent. Both postprandial hypertension and postprandial hypotension have been reported, but most of these data are collected in aging or patients ${ }^{29-31}$. As the metabolic disturbance following feeding is more complex than the glucose challenge, changes in postprandial BP in healthy individuals need to be further investigated. 


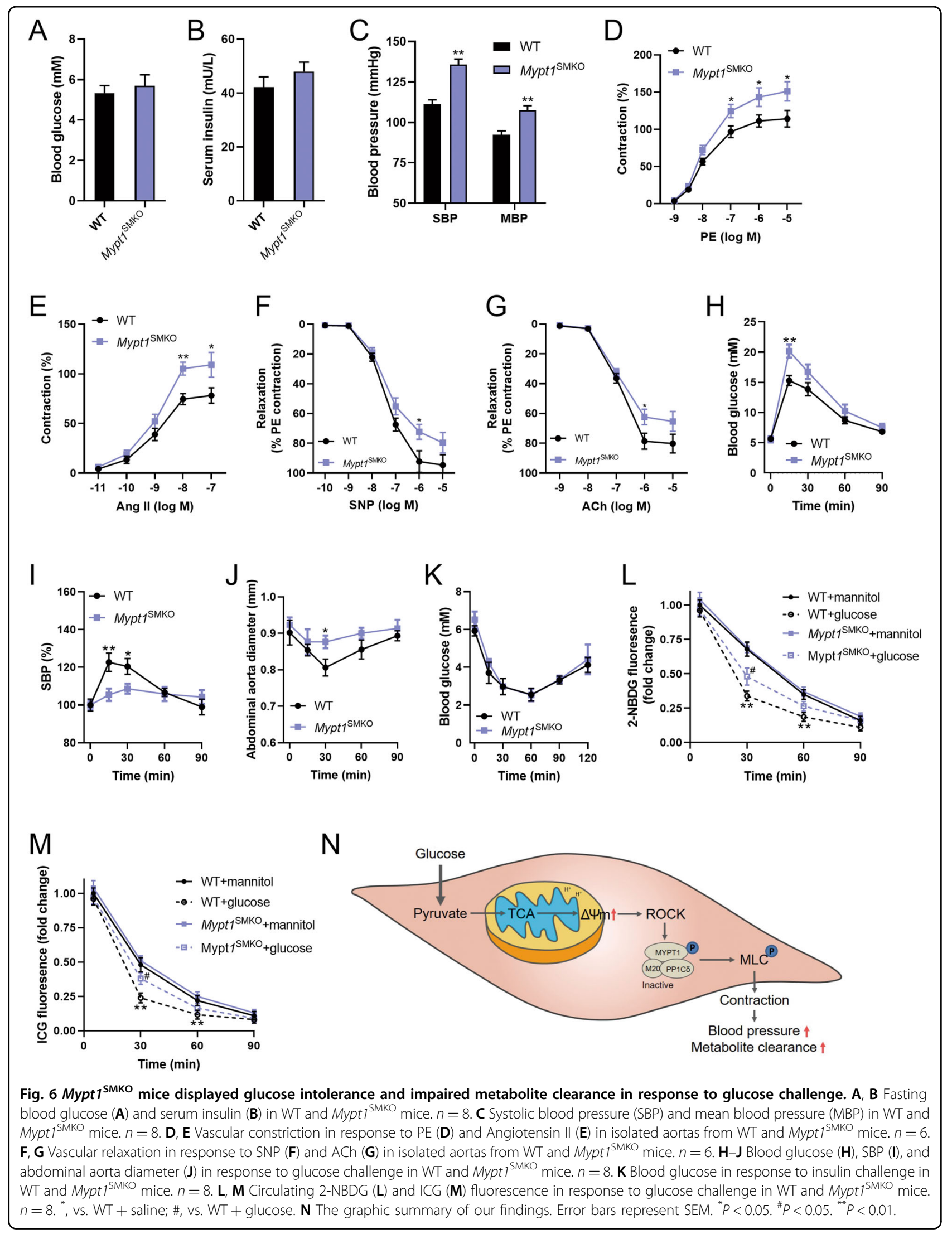


Different from striated muscle cells, VSMCs exhibit unusually high rates of glycolysis ${ }^{11}$. In spite of the low contribution of mitochondria to VSMC bioenergetics, evidence has shown that mitochondria widely regulate VSMC phenotype and function ${ }^{32-34}$. Our results extended these findings that mitochondrial oxidation promotes VSMC contraction. Interestingly, the process is not dependent on mitochondria-derived ATP, but dependent on $\Delta \Psi \mathrm{m}$ which positively regulates VSMC contraction as evidenced by a series of experiments. In fact, studies have shown that mitochondrion is the most sophisticated and dynamic responsive sensing system and depolarization or hyperpolarization of mitochondria plays a critical role in the regulation of various signals or processes, including reactive oxygen species, mitochondrial permeability, $\mathrm{Ca}^{2+}$, antiviral immunity, apoptosis, and aging ${ }^{35}$. Depolarization of $\Delta \Psi \mathrm{m}$ is also a basic characteristic of several mitochondrial events including mitochondrial flash, flicker, and oscillation, which serve as important signals in multiple physiological and pathological processes ${ }^{36-38}$. We found that mitochondrial hyperpolarization mediates the glucose influx-induced VSMC contraction, and inhi-

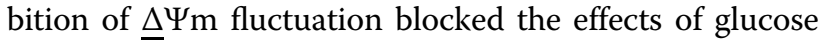
influx on the promotion of vascular constriction.

Smooth muscle tone is largely dependent on the relative activities of MLCK and MLCP ${ }^{39}$. As glucose influx has little effects on intracellular $\mathrm{Ca}^{2+}$ concentration and inhibition of intracellular $\mathrm{Ca}^{2+}$ fluctuation did not block the effects of glucose influx, glucose influx-induced VSMC contraction is not likely to be MLCK-mediated. Here, we found that glucose influx inhibited MLCP activity. Multiple signals have been reported to regulate MLCP activity in $\mathrm{VSMCs}^{39}$, among which ROCK is the major player. Glucose influx activated ROCK signaling and inhibition of ROCK signaling blocked the effects of glucose influx on VSMC contraction, indicating ROCK activation as a major step in $\Delta \Psi \mathrm{m}$-regulated smooth muscle tone. However, as the key molecule which activates ROCK signaling, RhoA is not involved in glucose influx-induced VSMC contraction as evidenced by the fact that glucose influx did not activate RhoA, and RhoA knockdown showed little effects on glucose influx-induced VSMC contraction. These results suggested that other small GTPases may be involved in the process. In fact, other Rho family proteins, including proteins from the Rho, Cdc42, Rac, and Rnd subfamilies, and even proteins from Ras superfamily of monomeric GTPases have been reported to be involved in the regulation of ROCK activity ${ }^{39,40}$, which should be tested in the future.

MLCP is mainly regulated by MYPT1 and CPI-17. Our data showed that both MYPT1 and CPI-17 are activated by mitochondrial hyperpolarization, in which MYPT1 is more responsive. Apart from protein kinase C, CPI-17 can also be activated by ROCK signaling ${ }^{41}$. MYPT1 is identified as an essential protein in the smooth muscle myosin phosphorylation module. MYPT1 enhances the catalytic activity and specificity of $\mathrm{PP} 1 \mathrm{c} \delta$ toward phosphorylated MLC in its binding to myosin. PP1c $\delta$ activity was inhibited by ROCK-induced phosphorylation of MYPT1 at Thr-696 or Thr-853, which has been shown to be either $\mathrm{Ca}^{2+}$. sensitive or $\mathrm{Ca}^{2+}$-independent ${ }^{17}$. Our data showed that $\Delta \Psi$ m-regulated MYPT1 activity was $\mathrm{Ca}^{2+}$-independent as evidenced by that modulation of $\mathrm{Ca}^{2+}$ signaling showed

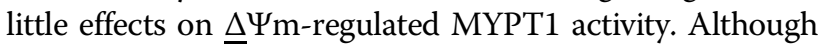
mounting evidence showed that MYPT1 played a central role in regulating MLCP activity and the phenotypic changes associated with adult Mypt1 ${ }^{\text {SMKO }}$ mice were modest, suggesting that MYPT1 is more likely to be a regulator of smooth muscle tone ${ }^{18,20}$. Here, we showed that knockout of MYPT1 in smooth muscle cells largely abolished the effects of both glucose and $\Delta \Psi \mathrm{m}$-regulated VSMC contraction in vivo and in vitro, indicating that MYPT1 plays a critical role in glucose influx-regulated VSMC contraction. Our results showed that besides MYPT1, there must be other regulators such as CPI-17 in the process. However, how $\Delta \Psi \mathrm{m}$ affects phosphorylation of MYPT1 has not been identified in the study. We tested the role of ROS in glucose influx-induced MYPT1 phosphorylation and found that cytosolic ROS was not likely to mediate $\Delta \Psi$ m-regulated cytosol protein phosphorylation, but mitochondrial ROS was involved in $\Delta \Psi$ m-regulated MYPT1 phosphorylation, suggesting that mitochondrial ROS may not need to be converted as cytosolic ROS to regulate cytosol protein phosphorylation. The underlying mechanism in mitochondrial ROS-regulated MYPT1 phosphorylation should be investigated in the future.

Taken together, our findings demonstrated that glucoseinduced VSMC contraction is mediated by mitochondrial hyperpolarization-activated MYPT1. $\Delta \Psi \mathrm{m}$ positively regulates MYPT1 activity which inhibits MLCP activity. This finding represents a novel mechanism underlying metabolic influx-induced vascular constriction and suggests that physiological blood glucose elevation contributes to physiological BP control and circulating metabolite clearance.

\section{Author details \\ ${ }^{1}$ School of Aerospace Medicine, Fourth Military Medical University, Xi'an 710032, China. ${ }^{2}$ Department of Cardiology, 986th Hospital, Fourth Military Medical University, Xi'an 710032, China. ${ }^{3}$ School of Basic Medical Sciences, Fourth Military Medical University, Xi'an 710032, China. ${ }^{4}$ Model Animal Research Center, Nanjing University, Nanjing 210061, China}

\section{Author contributions}

F.G. and X.Z. conceived and supervised the study, and wrote the manuscript. J.X., H.Y., Z.W., X.Q., J.Z., L.D., and L.Y. performed experiments. X.Z., J.X., J.L., and L.D. analyzed data. M.Z. provided knockout mice and conceived the study.

\section{Ethics statement}

The human study was approved by the Human Research Ethics Committee of Fourth Military Medical University. Animal experiments were approved by Fourth Military Medical University Committee on Animal Care. 


\section{Funding}

This work was supported by grants from the National Key Research and Development Program of China (2019YFF0301600) and the National Natural Science Foundation of China (Nos. 31871146, 81800360, 81670253, 31771265, 32071169, and 31971059).

\section{Conflict of interest}

The authors declare no competing interests.

\section{Publisher's note}

Springer Nature remains neutral with regard to jurisdictional claims in published maps and institutional affiliations.

Supplementary information The online version contains supplementary material available at https://doi.org/10.1038/s41419-021-03462-9.

Received: 16 October 2020 Revised: 14 January 2021 Accepted: 20 January 2021

Published online: 12 February 2021

\section{References}

1. Arnett, D. K. \& Claas, S. A. Omics of blood pressure and hypertension. Circ. Res 122, 1409-1419 (2018).

2. Padmanabhan, S. \& Joe, B. Towards precision medicine for hypertension: a review of genomic, epigenomic, and microbiomic effects on blood pressure in experimental rat models and humans. Physiol. Rev. 97, 1469-1528 (2017).

3. Saxton, S. N., Clark, B. J., Withers, S. B., Eringa, E. C. \& Heagerty, A. M. Mechanistic links between obesity, diabetes, and blood pressure: role of perivascular adipose tissue. Physiol. Rev. 99, 1701-1763 (2019).

4. Webb, R. C. Smooth muscle contraction and relaxation. Adv. Physiol. Educ. 27, 201-206 (2003).

5. Brozovich, F. V., Nicholson, C. J., Degen, C. V., Gao, Y. Z., Aggarwal, M. \& Morgan K. G. Mechanisms of vascular smooth muscle contraction and the basis for pharmacologic treatment of smooth muscle disorders. Pharm. Rev. 68 , 476-532 (2016).

6. Ford, T. J., Corcoran, D., Padmanabhan, S., Aman, A., Rocchiccioli, P. \& Good, R. et al. Genetic dysregulation of endothelin-1 is implicated in coronary microvascular dysfunction. Eur. Heart J. 41, 3239-3252 (2020).

7. Sutendra, G., Bonnet, S., Rochefort, G., Haromy, A., Folmes, K. D. \& Lopaschuk, G. D. et al. Fatty acid oxidation and malonyl-CoA decarboxylase in the vascular remodeling of pulmonary hypertension. Sci. Transl. Med. 2, 44 ra58 (2010).

8. Guo, Y., Wang, S., Liu, Y., Fan, L., Booz, G. W. \& Roman, R. J. et al. Accelerated cerebral vascular injury in diabetes is associated with vascular smooth muscle cell dysfunction. Geroscience 42, 547-561 (2020).

9. Wang, D. D., Toledo, E., Hruby, A., Rosner, B. A., Willett, W. C. \& Sun, Q. et al. Plasma ceramides, mediterranean diet, and incident cardiovascular disease in the PREDIMED Trial (Prevencion con Dieta Mediterranea). Circulation 135, 2028-2040 (2017).

10. Dunham-Snary, K. J., Wu, D., Potus, F., Sykes, E. A., Mewburn, J. D. \& Charles, R. L. et al. Ndufs2, a core subunit of mitochondrial complex i, is essential for acute oxygen-sensing and hypoxic pulmonary vasoconstriction. Circ. Res. 124, 1727-1746 (2019).

11. Butler, T. M. \& Siegman, M. J. High-energy phosphate metabolism in vascular smooth muscle. Annu. Rev. Physiol. 47, 629-643 (1985).

12. Paul, R. J. Functional compartmentalization of oxidative and glycolytic metabolism in vascular smooth muscle. Am. J. Physiol. 244, C399-409 (1983).

13. Chiong, M., Cartes-Saavedra, B., Norambuena-Soto, I., Mondaca-Ruff, D., Morales, P. E. \& Garcia-Miguel, M. et al. Mitochondrial metabolism and the control of vascular smooth muscle cell proliferation. Front Cell Dev. Biol. 2, 72 (2014).

14. Chalmers, S., Saunter, C., Wilson, C., Coats, P., Girkin, J. M. \& McCarron, J. G. Mitochondrial motility and vascular smooth muscle proliferation. Arterioscler Thromb. Vasc. Biol. 32, 3000-3011 (2012).

15. Salabei, J. K. \& Hill, B. G. Mitochondrial fission induced by platelet-derived growth factor regulates vascular smooth muscle cell bioenergetics and cell proliferation. Redox Biol. 1, 542-551 (2013).

16. Lincoln, T. M. Myosin phosphatase regulatory pathways: different functions or redundant functions? Circ. Res. 100, 10-12 (2007).
17. Dimopoulos, G. J., Semba, S., Kitazawa, K., Eto, M. \& Kitazawa, T. Ca ${ }^{2+}$-dependent rapid $\mathrm{Ca}^{2+}$ sensitization of contraction in arterial smooth muscle. Circ. Res 100, 121-129 (2007).

18. Qiao, Y. N., He, W. Q., Chen, C. P., Zhang, C. H., Zhao, W. \& Wang, P. et al. Myosin phosphatase target subunit 1 (MYPT1) regulates the contraction and relaxation of vascular smooth muscle and maintains blood pressure. J. Biol. Chem. 289, 22512-22523 (2014).

19. Huang, Q. F., Aparicio, L. S., Thijs, L., Wei, F. F., Melgarejo, J. D. \& Cheng, Y. B. et al. Cardiovascular end points and mortality are not closer associated with central than peripheral pulsatile blood pressure components. Hypertension 76, 350-358 (2020).

20. He, W. Q., Qiao, Y. N., Peng, Y. J., Zha, J. M., Zhang, C. H. \& Chen, C. et al. Altered contractile phenotypes of intestinal smooth muscle in mice deficient in myosin phosphatase target subunit 1. Gastroenterology 144, 1456-1465 (2013). 1465 e1451-1455.

21. Yang, L., Zhang, J., Xing, W., Zhang, X., Xu, J. \& Zhang, H. et al. SIRT3 deficiency induces endothelial insulin resistance and blunts endothelial-dependent vasorelaxation in mice and human with obesity. Sci. Rep. 6, 23366 (2016).

22. Yang, F., Chen, Q., He, S., Yang, M., Maguire, E. M. \& An, W. et al. miR-22 is a novel mediator of vascular smooth muscle cell phenotypic modulation and neointima formation. Circulation 137, 1824-1841 (2018).

23. Zhang, X., Xu, J., Cai, X., Ji, L., Li, J. \& Cao, B. et al. Acute insulin resistance mediated by advanced glycation endproducts in severely burned rats. Crit. Care Med 42, e472-480 (2014).

24. Johnson, L. V., Walsh, M. L., Bockus, B. J. \& Chen, L. B. Monitoring of relative mitochondrial membrane potential in living cells by fluorescence microscopy. J. Cell Biol. 88, 526-535 (1981).

25. Liao, J. K., Seto, M. \& Noma, K. Rho kinase (ROCK) inhibitors. J. Cardiovasc Pharm. 50, 17-24 (2007)

26. Wright, B. J., O'Brien, S., Hazi, A. \& Kent, S. Increased systolic blood pressure reactivity to acute stress is related with better self-reported health. Sci. Rep. $\mathbf{4}$ 6882 (2014)

27. Ayada, C., Toru, U. \& Korkut, Y. The relationship of stress and blood pressure effectors. Hippokratia 19, 99-108 (2015).

28. Syed, A. U., Reddy, G. R., Ghosh, D., Prada, M. P., Nystoriak, M. A. \& Morotti, S. et al. Adenylyl cyclase 5 -generated CAMP controls cerebral vascular reactivity during diabetic hyperglycemia. J. Clin. Invest. 129, 3140-3152 (2019).

29. Ward, K. A., DiPette, D. J., Held, T. N. \& Jain, R. K. Effect of intravenous versus intraperitoneal glucose injection on systemic hemodynamics and blood flow rate in normal and tumor tissues in rats. Cancer Res. 51, 3612-3616 (1991).

30. Huber, D. A., Carmo, J. M., Castania, J. A., Fazan, R. Jr. \& Salgado, H. C. Does acute hyperglycemia alter rat aortic depressor nerve function? Braz. J. Med Biol. Res. 40, 1567-1576 (2007).

31. Uetani, E., Tabara, Y., Igase, M., Guo, H., Kido, T. \& Ochi, N. et al. Postprandial hypertension, an overlooked risk marker for arteriosclerosis. Atherosclerosis 224, 500-505 (2012).

32. Yu, E. P., Bennett, M. R. Mitochondrial DNA damage and atherosclerosis. Trends Endocrinol. Metab. 25, 481-487 (2014).

33. Dasgupta, A., Wu, D., Tian, L., Xiong, P. Y., Dunham-Snary, K. J. \& Chen, K. H. et al. Mitochondria in the pulmonary vasculature in health and disease: oxygen-sensing, metabolism, and dynamics. Compr. Physiol. 10, 713-765 (2020).

34. Liu, Y. F., Zhu, J. J., Yu Tian, X., Liu, H., Zhang, T. \& Zhang, Y. P. et al. Hypermethylation of mitochondrial DNA in vascular smooth muscle cells impairs cell contractility. Cell Death Dis. 11, 35 (2020).

35. Dromparis, P. \& Michelakis, E. D. Mitochondria in vascular health and disease. Annu Rev. Physiol. 75, 95-126 (2013).

36. Wang, X., Zhang, X., Huang, Z., Wu, D., Liu, B. \& Zhang, R. et al. Protons trigger mitochondrial flashes. Biophys. J. 111, 386-394 (2016).

37. Chalmers, S., Saunter, C. D., Girkin, J. M. \& McCarron, J. G. Flicker-assisted localization microscopy reveals altered mitochondrial architecture in hypertension. Sci. Rep. 5, 16875 (2015).

38. Chalmers, S. \& McCarron, J. G. The mitochondrial membrane potential and $\mathrm{Ca}^{2+}$ oscillations in smooth muscle. J. Cell Sci. 121, 75-85 (2008).

39. Puetz, S., Lubomirov, L. T. \& Pfitzer, G. Regulation of smooth muscle contraction by small GTPases. Physiology (Bethesda) 24, 342-356 (2009).

40. Etienne-Manneville, S. \& Hall, A. Rho GTPases in cell biology. Nature $\mathbf{4 2 0}$ 629-635 (2002)

41. Koyama, M., Ito, M., Feng, J., Seko, T., Shiraki, K. \& Takase, K. et al. Phosphorylation of CPI-17, an inhibitory phosphoprotein of smooth muscle myosin phosphatase, by Rho-kinase. FEBS Lett. 475, 197-200 (2000). 